\title{
SETS EXPRESSIBLE AS UNIONS OF TWO CONVEX SETS
}

\author{
W. R. HARE, JR. AND JOHN W. KENELLY
}

A graph-theoretic formulation of McKinney's [1] characterization of the union of two convex sets leads to an especially concise proof, and suggests a technique which may prove quite useful in certain combinatorial-geometric problems. If $S$ is a set in a real linear space, we define the nonvisibility graph $G(S)$ of $S$ as the graph whose vertices are the points of $S$ and whose edges are defined by: if $x, y \in S$, then $x$ and $y$ are joined by an edge in $G(S)$ if and only if $x y \nsubseteq S$ (i.e., $x$ and $y$ are not visible in $S$ ). McKinney's property $P_{0}$ translates into the graph-theoretic condition that $G(S)$ has no circuits of odd length. It is a standard theorem (see [2]) that a graph has no circuits of odd length if and only if it is bipartite.

THeOREM. In a topological linear space a closed nonconvex set $S$ is expressible as the union of two convex sets if and only if $G(S)$ contains no circuits of odd length, i.e., if and only if $G(S)$ is bipartite.

Proof. The necessity of the condition on $G(S)$ is obvious, so only the proof of sufficiency of this condition is given.

Let the vertex set of $G(S)$ be written as $S=A \cup B$, where $A \cap B=\varnothing$, and where the only edges of $G(S)$ join points of $A$ with points of $B$. To prove the assertion, it suffices to prove that conv $A \cup \operatorname{conv} B \subset S$. In turn, by Carathéodory's Theorem, we need prove only the following assertion: if $\left\{a_{0}, \cdots, a_{k}\right\} \subset A$ is the vertex set of a $k$-simplex $T$, then $T \subset S$. The assertion is obvious if $k=0$ or $k=1$, so we assume that $k \geqq 2$ and that the statement is true for all subsets of $A$ having fewer than $k$ elements. In particular, we know that the boundary of $T$ is a subset of $S$. If there is a point $p$ which is relatively interior to $T$, but is not in $S$, we may as well assume that there is some open $k$-ball $U$ centered at $p$ such that $U$ is in the relative interior of $T$, but is disjoint from $S$.

By the decomposition of $S$ induced from $G(S)$, we have that the boundary of $T$ is decomposed into disjoint subsets with respect to $A$ and $B$. Each point $a$ of $\operatorname{bd} T$ which is an $A$ point can be mapped into a point of bd $T$ by extending the segment $a p$ to meet bd $T$ at the other point $t(a)$ distinct from $a$. This image point is necessarily a point $t(a)=b \in B$; for, since $a b \varangle S, a$ and $b$ are joined in $G(S)$. Now

Received by the editors August 9, 1968 and, in revised form, October 10, 1969. 
the cone formed by taking the union of all rays from $t(a)$ through points of $U$ meets bd $T$ in a neighborhood $N$ of $a$ and the preceding argument shows that $N \subset A \cap \mathrm{bd} T$. Hence, we have that the set of $A$ points in bd $T$ is open, and a similar argument gives that the set of $B$ points in $\operatorname{bd} T$ is also open. But these sets are disjoint and nonvoid; hence, $\operatorname{bd} T$ is not connected, a contradiction. This completes the proof.

Added in Proof. Richard L. McKinney has shown that the corresponding theorem for unions of three convex sets is valid. For unions of four or more convex sets, however, the analogous statements are false. (See Notices Amer. Math. Soc. 17 (1970), 245. Abstract \#672-575.)

\section{REFERENCES}

1. Richard L. McKinney, On unions of two convex sets, Canad. J. Math. 18 (1966), 883-886. MR 34 \#1923.

2. Oystein Ore, Theory of graphs, Amer. Math. Soc. Colloq. Publ., vol. 38, Amer. Math. Soc., Providence, R. I., 1962. MR 27 \#740.

Clemson University, Clemson, South Carolina 29631 